\title{
Fit of aggregation models to the distribution of group sizes in Northwest Atlantic seabirds
}

\author{
Guy Beauchamp* \\ Faculty of Veterinary Medicine, PO Box 5000, St-Hyacinthe, Québec J2S 7C6, Canada
}

\begin{abstract}
Many species of animals live in groups, but the mechanisms responsible for the formation and cohesion of groups are not well known and have not often been quantified. Different models of aggregations, all making unique assumptions about rates of arrivals to and departures from groups, produce different group size distributions. Fitting such models to empirical distributions of group sizes can provide insights into the mechanisms that underlie aggregations in animals. Four models predicting the distribution of group sizes (the power law, Poisson, exponential and negative binomial models) were tested in 7 species of Northwest Atlantic seabirds using maximum-likelihood estimation and a random factor to control for differences in mean group size among seabird surveys. Of the 4 models tested, the power law ranked first in most species in terms of raw Akaike's information criterion values. Nevertheless, likelihood-ratio tests revealed that the power law and the negative binomial model, which ranked second in most species, often provided a statistically similar fit to the data. Goodness-of-fit tests revealed a rather good fit of these models to the data. The finding that aggregation models can apply to the distribution of group sizes offers the opportunity to examine quantitatively the mechanisms or underlying processes that give rise to aggregations in seabirds and possibly other animals.
\end{abstract}

KEY WORDS: Seabirds · Power law · Maximum likelihood $\cdot$ Group size

\section{INTRODUCTION}

Many species of animals, ranging from invertebrates to birds and mammals, live in groups. Living in groups is thought to confer several adaptive benefits, such as increased foraging efficiency and protection against predators (Krause \& Ruxton 2002). While the costs and benefits of grouping are becoming increasingly clear, the mechanisms that regulate the formation and cohesion of groups are less well known. Krause \& Ruxton (2002) considered exploration of these mechanisms to be a key area of future research on group living. One approach thus far has been to test aggregation models that emphasize specific rules that individuals employ for joining and for leaving groups. Different sets of rules produce different group size distributions (Cohen 1972, Okubo 1986, Silverman et al. 2001), a feature that can then be used to discriminate among candidate processes that lead to the formation and dissolution of groups.
Several models have already been used to predict the size distribution of groups that form and dissolve rapidly. In such ephemeral groups, changes in group size are not related to long-term demographic factors such as birth and death, which are more relevant to groups with stable membership across most of the year, but reflect instead responses to more direct ecological factors. Earliest tests focused on Poisson and negative binomial distributions (Cohen 1972). In a Poisson distribution (see Appendix 1 for mathematical details), the frequency distribution of observed group sizes peaks at small group sizes and larger groups are rarely observed. With the negative binomial distribution, the variance in observed group sizes is larger and the distribution of observed group sizes is more heavily skewed to the right because larger groups are more likely to occur.

In terms of underlying ecological processes that can produce such distributions, both models assume that departures from a group over time increase linearly with group size. This assumption matches, for instance, 
the observation that aggression usually increases with group size because of increased competition (Caraco 1979). In contrast, the 2 models make different assumptions about the rate of arrivals to groups over time. The negative binomial model assumes that arrivals increase linearly with group size, mimicking the attraction that groups of animals have on nearby foragers (Pöysä 1992). For instance, individuals may be attracted to groups to reduce their predation risk or to find resources more quickly (Haney et al. 1992, Beauchamp et al. 1997, Davoren et al. 2003, Silverman et al. 2004). Arrivals are independent of group size in the Poisson model, and aggregation would only reflect independent arrivals to a site whose features, such as location for instance, are attractive regardless of the number of foragers present. Support for the Poisson model has been found in some bird and monkey species (Cohen 1975, Caraco 1980), and support for the negative binomial model has also been found in some fish, bird and monkey species (Cohen 1972, Wood 1985, O'Neill \& Faddy 2003).

Other models have been considered more recently. The power law implies that individuals aggregate when they meet and that groups split at a fixed rate independent of group size (Bonabeau et al. 1999). A key feature of the power law is that in a log-log plot, the relationship between the frequency of groups of different sizes and group size is a straight line with a negative slope, producing a scale-free parameter. The power law has been applied primarily to fish groups, but also to insects, birds and mammals (Sjoberg et al. 2000, Jovani et al. 2008). The exponential model pertains to situations when both the arrival and departure rates are independent of group size and mean group size is small (Bonabeau et al. 1999, Duerr \& Dietz 2000). This model has been applied to fish groups (Niwa 2003). With the exponential model, the relationship between the frequency of groups of different sizes and group size is not a straight line in a log-log plot, but is straight on a log-linear plot.

The above models make quantitatively different predictions regarding the shape of group size distributions. There are 4 issues related to fitting models to group size data to discriminate among such models. The first consideration should be to compare the fit of several models with the same dataset. Many reports have only tested a single model (e.g. Bonabeau et al. 1999, Sjoberg et al. 2000), and it is therefore difficult to evaluate the relevance of alternative models, which may actually provide a better fit and more biological insights (Brown et al. 2002). Second, the actual method of fitting models is also essential, since different methods produce different results. Maximum-likelihood estimation has been shown to be vastly more accurate than the ordinary least-squares method, especially when it comes to fitting exponents of the power law (Edwards 2008, White et al. 2008). However, such methods have not yet been applied to group size distributions. Third, comparing the fit of different models can be done using a likelihood-ratio test, to determine if one model provides a statistically better fit than another. Finally, the fit of the models should be tested statistically to determine how well predictions compare to empirical observations (Silverman et al. 2001, Clauset et al. 2009). This step is important because the model with the best fit in a set may still fit the data poorly.

To address these issues, I compared theoretical predictions to empirical group size distributions in 7 seabird species of the Northwest Atlantic. Surveys in this area are conducted frequently and provide a large number of sightings to test these models. I used maximum-likelihood estimation and compared models using likelihood-ratio tests. Goodness-of-fit tests were then used to compare predicted and observed values.

Fitting a particular model to the data represents a methodological challenge, but this is more than a fitting exercise, because it can provide biological insights and help to identify ecological processes. The set of rules that underlie a particular distribution of group sizes can shed light on the mechanisms used by animals to join and leave groups. Ultimately, a better understanding of these mechanisms will be useful to evaluate the reasons why individuals live in groups.

\section{MATERIALS AND METHODS}

The Eastern Canada Seabirds at Sea (ECSAS) survey database of the Canadian Wildlife Service, Environment Canada, was used. Survey data have been collected in the Northwest Atlantic since the 1970s, but recently a more standard protocol has been used to collect group size data. Survey results from this area have been used earlier to estimate population densities and to address ecological questions (e.g. Huettmann \& Diamond 2001, 2006).

The area covered by the surveys ranges from 40 to $60^{\circ} \mathrm{N}$ and 70 to $41^{\circ} \mathrm{W}$. During surveys from 2006 to 2009, group sizes of flying birds and of birds on the water were gathered systematically within $300 \mathrm{~m}$ of the ship every 5 to $10 \mathrm{~min}$. Of particular importance is the distance at which numbers of birds are gathered, since the ability to detect seabirds, especially the smaller species, is known to decline quite rapidly beyond 300 m (Barbraud \& Thiebot 2009).

In these surveys, the activities of the birds on the water have not been noted systematically; thus, it is not known if birds aggregated over food resources or were simply resting. In addition to the number of birds present, the dataset contains information about time of day 
and provides the geographical location of the sampling point at the beginning of each 5 to 10 min interval. Encountering a flock is quite a rare event, and most 5 to 10 min bins are empty. A flock was defined as an aggregation of 2 or more birds flying or occurring together on the water, generally within meters of one another.

The analyses were restricted to the 7 most common seabird species recorded during winter surveys, thus avoiding major fluctuations in group sizes related to breeding and migration. Sample size for other species was rather small and provided too little power for analysis. The 7 most common seabird species in the survey data were black-legged kittiwake (BLKI, Rissa tridactyla), dovekie (DOVE, Alle alle), great black-backed gull (GBBG, Larus marinus), greater shearwater (GRSH, Puffinus gravis), herring gull (HERG, Larus argentatus), northern fulmar (NOFU, Fulmarus glacialis) and thick-billed murre (TBMU, Uria lomvia).

Four different truncated models were tested using maximum-likelihood estimation. Truncation refers to the fact that groups of size zero are not recorded. These models are described in Appendix 1. Each model was fitted separately for each species and for each type of group (flying or on the water) for a total of 14 species/ group type combinations. A random effect was included in each model associated with the specific cruise. Because survey cruises differed in length and occurred at different times in the season or in different years, mean group size is expected to vary from one survey to another, and the random factor was used to model this extrinsic variability.

Maximum-likelihood estimation requires determination of the log-likelihood function for each model. The goal is essentially to determine the likelihood of a value for the unknown parameter of a model given the data. Log-likelihood functions for each model were obtained from the literature (Grogger \& Carson 1991, Clauset et al. 2009). For a given species/group type, the log-likelihood is calculated over all sightings, and maximum-likelihood estimation provides an estimate for each parameter underlying each model. Given that a random variable was used, the solution provides parameter estimates for each survey cruise. The procedure Nlmixed from SAS V. 9.1 was used.

To determine whether a model provided a better fit of the data than another, the 2 models that were ranked first and second in terms of raw values of Akaike's information criterion (AIC) were compared. The other 2 models were less likely, as shown below, and were not tested against the top models. The likelihood-ratio test allows one to determine if the difference between the fit of 2 models is statistically significant. A test was performed for each species and for each group type separately. The first step was to compute the difference between the log-likelihood values calculated from each model for each sighting. These log-likelihood values were obtained using the parameters fitted for each model (Table 1). The cumulative difference, or deviance, was then obtained across all sightings. A p-value for the test was obtained using Eq. (C-6) from Clauset et al. (2009) and took into account the expected variance for the deviance and the total number of sightings. A p-value $<0.05$ indicated that the deviance was not likely due to chance alone and that we can trust that one model provided a better fit to the data than the alternative.

A goodness-of-fit test provides an estimate of the quality of the fit of a model to the data. Here, I examined the fit of the power law, which ranked first in most cases (see 'Results'), for each species and for each group type separately. To increase power, I pooled data across all censuses. I used the $G$-test for goodness-of-fit to evaluate fit in each case. The first step was to calculate the $G$-test statistic value for the observed data (Sokal \& Rohlf 1995). The G-test for goodness-of-fit compares the observed frequencies for all group sizes recorded to the predicted frequencies obtained from the power law. To generate the predicted frequencies of group sizes, the estimated parameter values from the maximum-likelihood procedure described above (but for all sightings) were plugged into the power law's probability density function (pdf) (Appendix 1). The pdf generates predicted probabilities of occurrence of groups of any size. These probabilities were multiplied by the total number of sightings to obtain predicted frequencies of groups of any size.

The second step was to obtain a p-value for the $G$ test statistic value. I used a Monte Carlo approach as detailed in Clauset et al. (2009). The basic approach is to generate $1000 \mathrm{G}$-test statistic values using synthetic distributions of group sizes and to determine whether the $G$-test statistic value for the observed data falls below the 95th percentile of the generated $G$-test statistic values $(p>0.05)$. When this occurs, the model provides a good fit to the data.

The procedure to generate synthetic group size data is illustrated for 1 species with $n$ sightings. A total of $n$ group size values was drawn randomly from a power law distribution with the fitted parameter for that species. This simulated distribution of group sizes was then compared to the predicted values generated using the power law distribution fitted to this particular simulated distribution. A $G$-test statistic value for this comparison was then calculated just as was the case for the empirical data. This procedure was repeated 1000 times to generate 1000 synthetic $G$-test statistic values.

For species where the power law fitted more poorly than alternative models, I also ran goodness-of-fit tests using the same procedure as above, but using the pdf of the alternative model. 


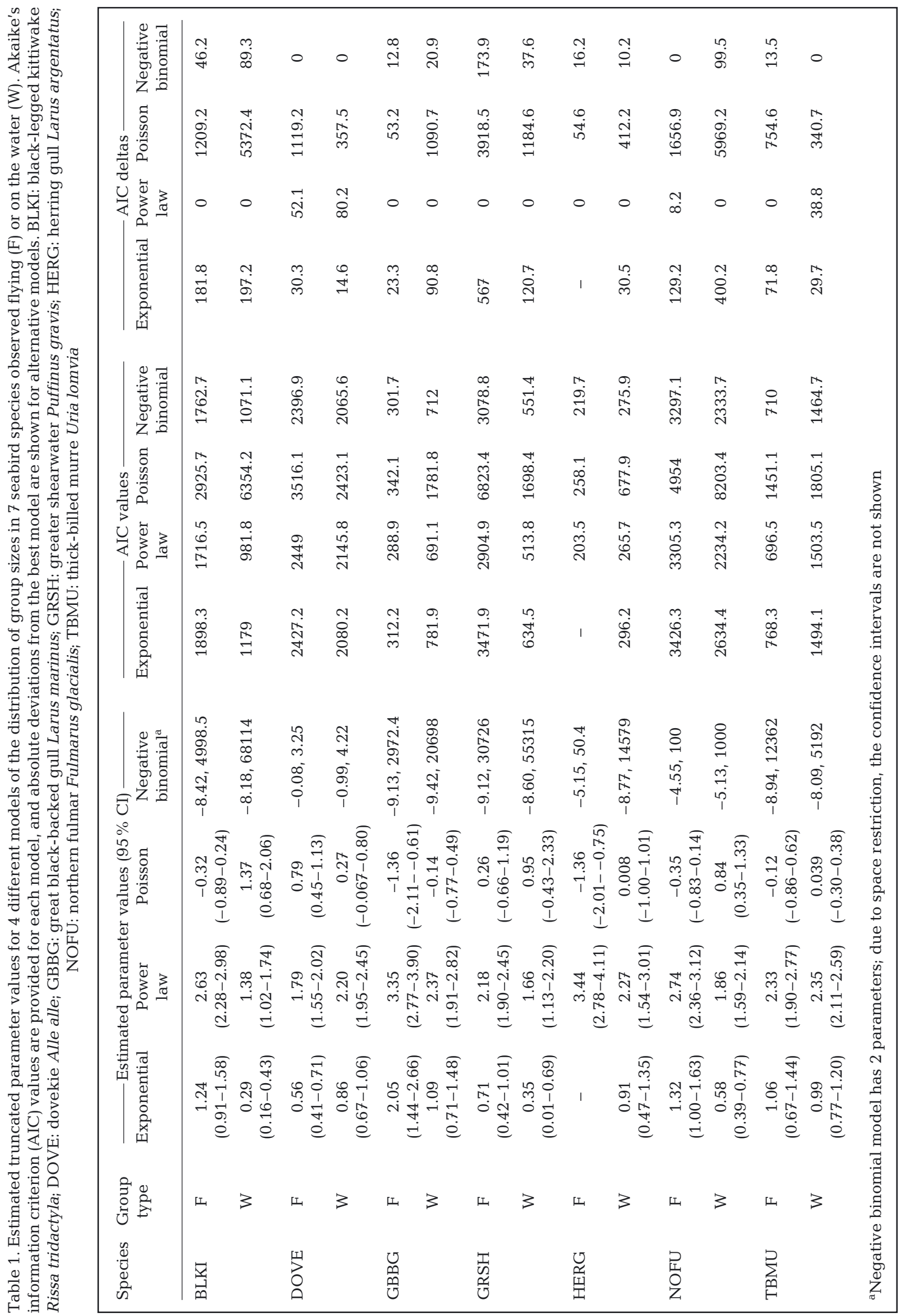




\section{RESULTS}

The dataset consisted of 38 winter survey cruises, with a median transect length of $238 \mathrm{~km}$ (range: 13 to $1732 \mathrm{~km}$ ). Total number of sightings per species ranged from 288 to 1801, with a median of 974 . Frequencies of group sizes generally decreased with group size for each species and for each type of group (Fig. 1).

Table 1 provides the fitted parameters for all models and their raw AIC values. In terms of AIC values, the power law ranked first or second for all species/group type combinations. The negative binomial model was ranked first for dovekies Alle alle and thickbilled murres Uria lomvia on the water. Raw AIC values for the exponential and Poisson models were often $>100$ units away from the top models and are unlikely candidates to fit the data.

The power law provided a statistically better fit than the negative binomial in 4 species/group type combinations and a fit that was no better in 7 other combinations (Table 2). The negative binomial model provided a statistically better fit than the power law in dovekies Alle alle and thick-billed murres Uria lomvia on the water.
Table 2. Likelihood ratio tests between the power law and the negative binomial model for the distribution of group sizes for seabirds on the water (W) and flying (F) (see Table 1 for definition of species acronyms)

\begin{tabular}{|lcrll|}
\hline \multirow{2}{*}{ Species } & Group type & $\mathrm{N}$ & $\mathrm{p}$ & Interpretation \\
\hline \multirow{2}{*}{ BLKI } & $\mathrm{F}$ & 188 & 0.63 & Equal fit \\
& $\mathrm{W}$ & 786 & 0.002 & Power law is a better fit \\
DOVE & $\mathrm{F}$ & 587 & 0.02 & Power law is a poorer fit \\
& $\mathrm{W}$ & 809 & 0.002 & Power law is a poorer fit \\
GBBG & $\mathrm{F}$ & 253 & 0.49 & Equal fit \\
& $\mathrm{W}$ & 211 & 0.87 & Equal fit \\
GRSH & $\mathrm{F}$ & 1006 & 0.006 & Power law is a better fit \\
& $\mathrm{W}$ & 113 & 0.04 & Power law is a better fit \\
HERG & $\mathrm{F}$ & 203 & 0.82 & Equal fit \\
& $\mathrm{W}$ & 85 & 0.84 & Equal fit \\
NOFU & $\mathrm{F}$ & 1280 & 0.50 & Equal fit \\
& $\mathrm{W}$ & 521 & 0.004 & Power law is a better fit \\
TBMU & $\mathrm{F}$ & 235 & 0.83 & Equal fit \\
& $\mathrm{W}$ & 599 & 0.02 & Power law is a poorer fit \\
\hline
\end{tabular}

For those species/group type combinations where the power law provided an equal or better fit than the negative binomial model, the $G$-test for goodness-of-fit indicated a good fit of the model to the data (Table 3). In the 5 cases where the power law did not fit the data well, the negative binomial model also failed to provide a good fit (Table 3 ).
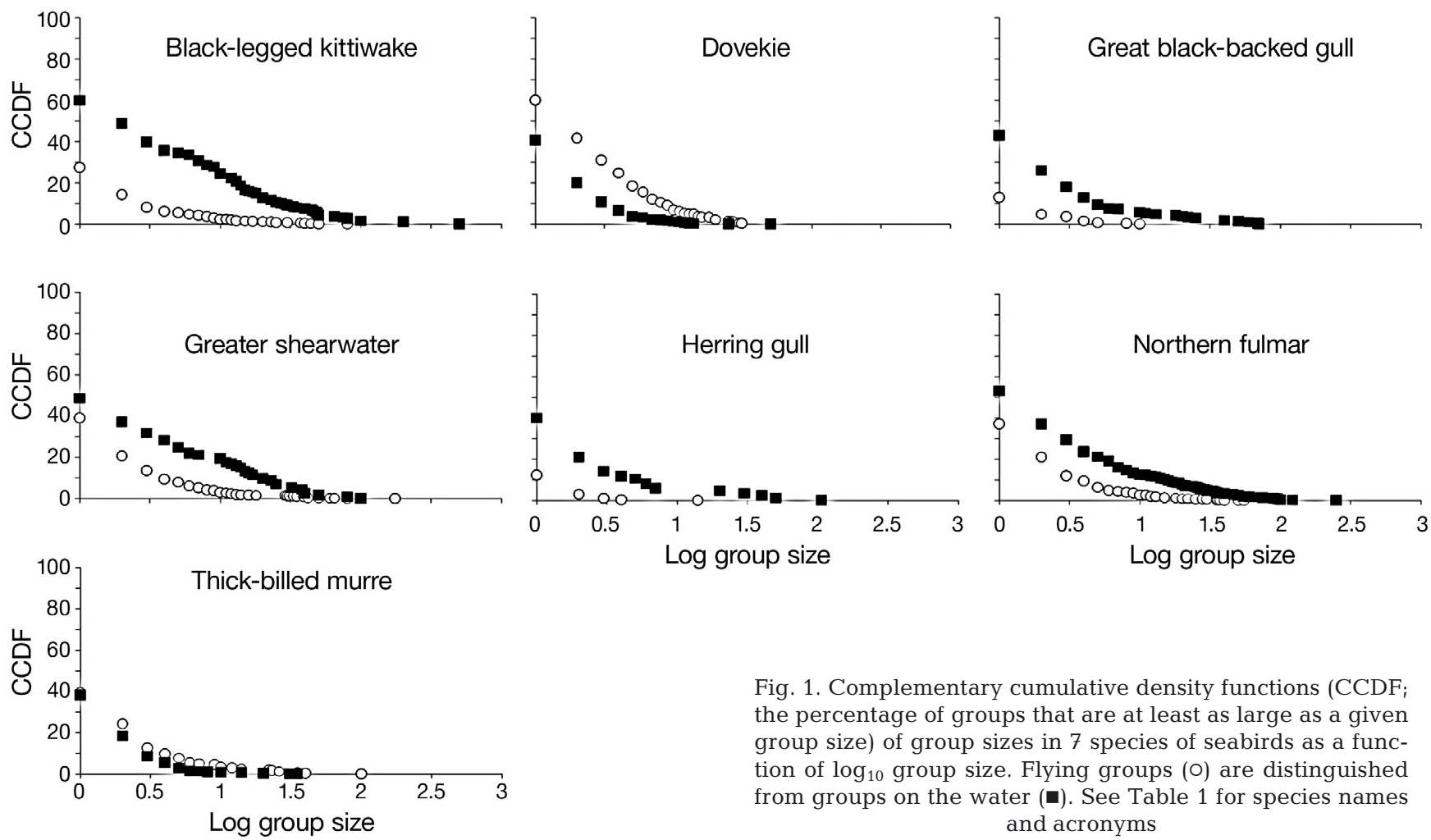

Fig. 1. Complementary cumulative density functions $(\mathrm{CCDF}$; the percentage of groups that are at least as large as a given group size) of group sizes in 7 species of seabirds as a function of $\log _{10}$ group size. Flying groups (O) are distinguished from groups on the water $(\varpi)$. See Table 1 for species names and acronyms 
Table 3. G-test for goodness-of-fit for the distribution of group sizes for seabirds on the water (W) and flying (F) (see Table 1 for definition of species acronyms)

\begin{tabular}{|c|c|c|c|c|c|c|}
\hline \multirow[t]{2}{*}{ Species } & \multirow[t]{2}{*}{ Group type } & \multirow[t]{2}{*}{$\mathrm{N}$} & \multicolumn{2}{|c|}{ Power law fit } & \multicolumn{2}{|c|}{ Negative binomial fit } \\
\hline & & & G-test & $\mathrm{p}$ & G-test & $\mathrm{p}$ \\
\hline \multirow[t]{2}{*}{ BLKI } & $\mathrm{F}$ & 786 & 14.3 & 0.15 & & \\
\hline & $\mathrm{W}$ & 188 & 40.5 & 0.02 & 57.0 & $<0.001$ \\
\hline \multirow[t]{2}{*}{ DOVE } & $\mathrm{F}$ & 587 & 44.8 & $<0.001$ & 10.9 & 0.003 \\
\hline & $\mathrm{W}$ & 809 & 62.2 & $<0.001$ & 24.4 & 0.003 \\
\hline \multirow[t]{2}{*}{ GBBG } & $\mathrm{F}$ & 253 & 11.8 & 0.16 & & \\
\hline & W & 211 & 12.9 & 0.20 & & \\
\hline \multirow[t]{2}{*}{ GRSH } & $\mathrm{F}$ & 1006 & 17.9 & 0.05 & & \\
\hline & W & 113 & 17.4 & 0.06 & & \\
\hline \multirow[t]{2}{*}{ HERG } & $\mathrm{F}$ & 203 & 5.4 & 0.71 & & \\
\hline & W & 85 & 7.4 & 0.63 & & \\
\hline \multirow[t]{2}{*}{ NOFU } & $\mathrm{F}$ & 1280 & 42.2 & $<0.001$ & 127.8 & $<0.001$ \\
\hline & $\mathrm{W}$ & 521 & 9.5 & 0.07 & & \\
\hline \multirow[t]{2}{*}{ TBMU } & $\mathrm{F}$ & 235 & 15.5 & 0.13 & & \\
\hline & $\mathrm{W}$ & 599 & 53.6 & $<0.001$ & 20.0 & 0.015 \\
\hline
\end{tabular}

\section{DISCUSSION}

The results indicate that for most of the seabird species examined here the truncated power law ranked first among the models tested. Nevertheless, the negative binomial model and power law provided a statistically similar fit to the data in many species. The G-test for goodness-of-fit indicated that the fit of these models to empirical data was quite good in most species. The results stress the importance of comparing several models before concluding that a single, specific model fits the data.

It is difficult to compare the specific parameter values obtained for each model with other empirical data since few earlier studies tested more than one model and often did not use maximum likelihood to fit the data. Nevertheless, I note that the scaling exponent for the power law varied widely among seabird species, from 1.3 to 3.4 , suggesting that a universal law is unlikely.

I now examine the consequences of fitting the power and negative binomial model to group size data. A fit to the power law suggests that individuals aggregate upon encounter, irrespective of group size, and leave groups at a fixed rate, also independently of group size. This is in sharp contrast to the assumption of group size-dependent departures in the negative binomial model. The negative binomial model further assumes that the probability of joining a group increases with group size. Since I could not discriminate between the power law and the negative binomial model in all species, more data are required to determine the effect of group size on arrivals to and departures from groups in seabird species. It is a sobering thought that with nearly 1000 sightings per species, it is still difficult to distin- guish between aggregation models that assume strikingly different patterns for joining and leaving groups.

Aggregations in seabirds are common for foraging and resting (e.g. Hoffman et al. 1981, Schneider \& Duffy 1985, Piatt 1990). Nevertheless, the effect of group size on the probability of joining or leaving groups is not clear. In common mergansers Mergus merganser, individual birds were more likely to join larger groups, but the power law was not tested (Wood 1985). The power law may not hold as well in strictly foraging groups, where competition for resources can occur (e.g. Lewis et al. 2001, Davoren et al. 2003) and where frequency-dependent departures are more likely. Because the activity of the birds on the water could not be determined, it is not clear what role competition for resources played in deviation from power law behaviour.

The power law or negative binomial model poorly fitted the data in some species/group type combinations. One possible explanation is visibility. In rough seas, counts of seabirds may be less accurate, especially for smaller species or for those in small groups, which may be difficult to see at a distance. Therefore, the observed distribution of group sizes may be biased towards larger groups in such cases. It would be interesting to see if this bias can account for the fact that the power law was less supported in dovekies, the smallest species in the study set.

Another problem is that the G-test for goodness-offit was performed by pooling results from all surveys. While pooling increased sample size and potentially power, it may also reduce fit by aggregating data encompassing significant ecological variation. For instance, group size frequencies in each individual census may follow one law (particular parameter values for a particular model), but if mean group size varies from one census to another, the summed frequencies over many censuses may fit another law, thus masking the real dynamics of group formation (Caraco 1980, Allen et al. 2001). This has been generally ignored in previous research. To minimize such problems when comparing the fit of different models, I restricted the analyses to a relatively stable part of the year in terms of population density and I used a random factor to account for differences among surveys in mean group size. Unfortunately, I could not use goodness-of-fit tests for each census, because the power of such testing is low when the sample size is small, as was the case for many censuses. 
Perhaps one of the most interesting consequences of fitting a particular aggregation model to group size data is that changes in parameter values may be indicative of biologically relevant changes in group formation dynamics. These shifts have been related to density-dependent factors, such as food availability and competition (Bonabeau et al. 1999, Sjoberg et al. 2000, Lusseau et al. 2004, Jovani et al. 2008). For instance, in stable populations of dolphins Tursiops truncatus, variation in food density was related to group size. The tendency to live in groups of different sizes when ecological conditions varied was detected using variation in the exponent of the power law (Lusseau et al. 2004). Variation in the value of the power law exponent or in the value of the negative binomial parameters could also be used in wintering seabirds to examine responses to any factor, such as changes in food availability caused by climate change or fishing for instance, which can influence group size preferences.

Future research could extend both the range of species tested and the number of models fitted to the data to seek trends among species that can be related to ecological factors such as diet and body size. In the present study, I restricted the analysis to mathematical models with ecological underpinnings, but other longtailed models could also be tested. The finding that aggregation models apply to the distribution of group sizes offers the opportunity to examine the mechanisms or processes that give rise to the emergent outcome of aggregations in seabirds and possibly other animals. A better understanding of the mechanisms underlying aggregations will shed more light on the adaptive value of group living and on the factors influencing the tremendous variation in group sizes that exist in animals.

Acknowledgements. I thank Carina Gjerdrum, with the Canadian Wildlife Service, for querying the ECSAS dataset for me and for comments on the paper. I also thank F. Huettmann and an anonymous referee for constructive comments on an earlier draft of the manuscript.

\section{LITERATURE CITED}

Allen AP, Li BL, Charnov EL (2001) Population fluctuations, power laws and mixture of lognormal distributions. Ecol Lett 4:1-3

Barbraud C, Thiebot JB (2009) On the importance of estimating detection probabilities from at-sea surveys of flying seabirds. J Avian Biol 40:584-590

- Beauchamp G, Bélisle M, Giraldeau LA (1997) Influence of conspecific attraction on the spatial distribution of learning foragers in a patchy habitat. J Anim Ecol 66:671-682

Bonabeau E, Dagorn L, Fréon P (1999) Scaling in animal group-size distributions. Proc Natl Acad Sci USA 96: $4472-4477$
Brown JH, Gupta VK, Li BL, Milne BT, Restrepo C, West GB (2002) The fractal nature of nature: power laws, ecological complexity and biodiversity. Phil Trans R Soc Lond Ser B 357:619-626

Caraco T (1979) Time budgeting and group size: a theory. Ecology 60:611-617

> Caraco T (1980) Stochastic dynamics of avian foraging flocks. Am Nat 115:262-275

> Clauset A, Shalizi CR, Newman MEJ (2009) Power-law distributions in empirical data. SIAM Rev 51:661-703

> Cohen JE (1972) Markov population processes as models of primate social and population dynamics. Theor Popul Biol 3:119-134

> Cohen JE (1975) The size and demographic composition of social groups of wild orang-utangs. Anim Behav 23: $543-550$

Davoren GK, Montevecchi WA, Anderson JT (2003) Distributional patterns of a marine bird and its prey: habitat selection based on prey and conspecific behaviour. Mar Ecol Prog Ser 256:229-242

> Duerr HP, Dietz K (2000) Stochastic models for aggregation processes. Math Biosci 165:135-145

> Edwards AM (2008) Using likelihood to test for Lévy flight search patterns and for general power-law distributions in nature. J Anim Ecol 77:1212-1222

Grogger JT, Carson RT (1991) Models for truncated counts. J Appl Econ 6:225-238

Haney JC, Fristrup KM, Lee DS (1992) Geometry of visual recruitment by seabirds to ephemeral foraging flocks. Ornis Scand 23:49-62

Hoffman W, Heinemann D, Wiens JA (1981) The ecology of seabird feeding flocks in Alaska. Auk 98:437-456

Huettmann F, Diamond AW (2001) Seabird colony locations and environmental determination of seabird distribution: a spatially-explicit breeding seabird model for the Northwest Atlantic. Ecol Model 141:261-298

Huettmann F, Diamond AW (2006) Large-scale effects on the spatial distribution of seabirds in the Northwest Atlantic. Landscape Ecol 21:1089-1108

Jovani R, Serrano D, Ursua E, Tella JL (2008) Truncated power laws reveal a link between low-level behavioural processes and grouping patterns in a colonial bird. PLoS One 3:e1992

Krause J, Ruxton GD (2002) Living in groups. Oxford University Press, Oxford

Lewis S, Sherratt TN, Hamer KC, Wanless S (2001) Evidence of intra-specific competition for food in a pelagic seabird. Nature 412:816-819

> Lusseau D, Williams R, Wilson B, Grellier K, Barton TR, Hammond PS, Thompson PM (2004) Parallel influence of climate on the behaviour of Pacific killer whales and Atlantic bottlenose dolphins. Ecol Lett 7:1068-1076

> Niwa HS (2003) Power-law versus exponential distributions of animal group sizes. J Theor Biol 224:451-457

> Okubo A (1986) Dynamic aspects of animal grouping: swarms, schools, flocks, and herds. Adv Biophys 22:1-94

> O'Neill MF, Faddy MJ (2003) Use of binary and truncated negative binomial modelling in the analysis of recreational catch data. Fish Res 60:471-477

Piatt JF (1990) The aggregative response of common murres and Atlantic puffins to schools of capelin. Stud Avian Biol 14:36-51

Pöysä H (1992) Group foraging in patchy environments: the importance of coarse-level local enhancement. Ornis Scand 23:159-166

> Schneider DC, Duffy DC (1985) Scale-dependent variability in seabird abundance. Mar Ecol Prog Ser 25:211-218 
Silverman ED, Kot M, Thompson E (2001) Testing a simple stochastic model for the dynamics of waterfowl aggregations. Oecologia 128:608-617

Silverman ED, Veit RR, Nevitt GA (2004) Nearest neighbors as foraging cues: information transfer in a patchy environment. Mar Ecol Prog Ser 277:25-35

Sjoberg M, Albrectsen B, Hjalten J (2000) Truncated power law: a tool for understanding aggregation patterns in animals? Ecol Lett 3:90-94
Sokal RR, Rohlf FJ (1995) Biometry. WH Freeman, New York, NY

White EP, Enquist BJ, Green JL (2008) On estimating the exponent of power-law frequency distributions. Ecology 89:905-912

> Wood CC (1985) Aggregative response of common mergansers (Mergus merganser): predicting flock size and abundance on Vancouver Island salmon streams. Can J Fish Aquat Sci 42:1259-1271

Appendix 1. Group size models

Four models were used to fit the group-size data. For a given species and for a given group type, the dataset consists of a sequence of sightings where group size is $\geq 1$, since groups of size 0 are not recorded. Probability density functions (pdf) are provided here for each model. The pdf determines the probability of observing a group of any particular size. In each model, there are $i=1,2, \ldots, n$ observations, $Y_{i}$ is the $i$ th observation and $j=1,2, \ldots$ are the possible values for $Y_{i}$.

\section{Truncated Poisson model}

The model has 1 parameter $\lambda$ (Grogger \& Carson 1991). The pdf is given by:

$$
\operatorname{Pr}\left(Y_{i}=j \mid Y_{i}>0\right)=\lambda^{j} /[\exp (\lambda)-1] j !
$$

Truncated negative binomial model

The model has 2 parameters $\alpha$ and $\lambda$ (Grogger $\&$ Carson 1991) and includes a gamma-function $(\Gamma)$. The pdf is given by:

$\operatorname{Pr}\left(Y_{i}=j \mid Y_{i}>0\right)=\{\Gamma(j+1 / \alpha) / \Gamma(j+1) \Gamma(1 / \alpha)\}(\alpha \lambda)^{j}(1+\alpha \lambda)^{-(j+1 / \alpha)}[1-F(0)]^{-1}$

\section{Truncated exponential}

The model has 1 parameter $\lambda$ (Clauset et al. 2009). The pdf is given by:

$$
\operatorname{Pr}\left(Y_{i}=j \mid Y_{i}>0\right)=\exp (-\lambda j)[\exp (\lambda)-1]
$$

\section{Truncated power law}

The model has 1 parameter $\alpha$ (Clauset et al. 2009). The pdf is given by:

$$
\operatorname{Pr}\left(Y_{i}=j \mid Y_{i}>0\right)=j^{-\alpha} /[\zeta(\alpha, 1)]
$$

where $\varsigma(\alpha, 1)=\Sigma(k+1)^{-\alpha}$ and the summation for $k$ is taken from 0 to infinity.

Editorial responsibility: Rory Wilson,

Swansea, UK
Submitted: September 30, 2010; Accepted: January 5, 2011

Proofs received from author(s): February 24, 2011 